\title{
ATRASOS NOS ENCAMINHAMENTOS DE PACIENTES COM SUSPEITA DE CÂNCER BUCAL: PERCEPÇÃO DOS CIRURGIÕES-DENTISTAS NA ATENÇÃO PRIMÁRIA À SAÚDE
}

\author{
Delay in Referral of Patients Suspected of Oral Cancer: Perception of Surgeon Dentists in Primary Health Care
}

Amanda Ramos da Cunha' ${ }^{1}$, Caren Serra Bavarescoㄹ, Vinícius Coelho Carrard ${ }^{3}$, Eduardo Madruga Lombardo 4

Resumo Objetivos: Verificar quais as possíveis razões que justifiquem o atraso da chegada do paciente com câncer bucal aos setores de maior complexidade de atenção em saúde. Materiais e métodos: $O$ estudo teve como público-alvo cirurgiões-dentistas atuantes em Atenção Primária à Saúde formalmente vinculados ao Projeto TelessaúdeRS (276 potenciais participantes). Foi utilizado um questionário hospedado em software de questionários online, com o link para acesso disponibilizado por correspondência eletrônica e através da homepage do TelessaúdeRS. As respostas foram tabuladas em uma planilha de Excel (Microsoft ${ }^{\oplus}$ ). Resultados: Setenta e quatro questionários respondidos (26,8\% da amostra inicial); $52,7 \%$ relatam não haver Centro de Especialidades Odontológicas para referência na sua Coordenadoria Regional de Saúde; 58,1\% consideram como possíveis causas do atraso no diagnóstico de câncer bucal a associação dos fatores: falha do profissional, do paciente, da rede de saúde e falta de informação; em relação à autopercepção de aptidão para rastreamento e diagnóstico de câncer bucal, $71,6 \%$ responderam que seriam importantes capacitações. Conclusões: $O$ presente estudo sugere como possíveis razões que justifiquem o atraso da chegada do paciente com câncer bucal aos setores de maior complexidade de atenção em saúde a deficiência da rede de atenção e a necessidade de mais capacitações relacionadas à patologia. Palavras-chave: Câncer Bucal; Diagnóstico Tardio; Atenção Primária à Saúde.

Abstract Aims: To verify the possible reasons to justify the delay in the arrival of the patient with oral cancer to the most complex sectors of health care. Materials and Methods: The study had as target audience dentists working in Primary Health Care formally linked to the Project TelessaúdeRS (276 potential participants). It was utilized a questionnaire hosted in a software of online questionnaires, with the link to access available by electronic mail and homepage of TelessaúdeRS. The responses were tabulated in an Excel spreadsheet (Microsoft $\left.{ }^{\circledR}\right)$. Results: Seventy-four answered questionnaires (26.8\% of the initial sample); $52.7 \%$ reported that there is no Dental Specialty Center for reference in its Regional Health's Coordination; $58.1 \%$ considered as the possible causes of the delay in diagnosis of oral cancer the association of factors: failure from professional, from patient, from the health network and lack of information; related to the self-perception of suitability for screening and diagnosis of oral cancer, $71.6 \%$ replied that it would be important the offer of qualification courses. Conclusion: The present study suggests that the possible reasons to justify the delay of the arrival of the patient with oral cancer to the most complex sectors of health care are: the deficiencies in the network care and the need for more qualifications courses related to the pathology.

Keywords: Mouth Neoplasms; Delayed Diagnosis; Primary Health Care.

1. Especialista em Saúde da Família e Comunidade. Cirurgiã-dentista da Associação Hospitalar Moinhos de Vento, Porto Alegre-RS/Brasil; 2. Pós-Doutoranda em Epidemiologia. Cirurgiã-dentista do Grupo Hospitalar Conceição. Teleconsultora e Telerreguladora do Projeto TelessaúdeRS, Porto Alegre-RS/Brasil; 3. Doutor em Odontologia (Patologia Bucal). Professor de Patologia, Faculdade de Odontologia - Universidade Federal do Rio Grande do Sul. Teleconsultor do Projeto TelessaúdeRS, Porto Alegre-RS/Brasil; 4. Especialista em Saúde da Família e Comunidade. Residente em Cirurgia e Traumatologia Bucomaxilofaciais pela Pontifícia Universidade Católica do Rio Grande do Sul, Porto Alegre-RS/Brasil 


\section{Introdução}

O câncer pode ser definido como um crescimento desordenado de células em pacientes suscetíveis, resultante do desequilíbrio entre divisão e apoptose celular'. O câncer de boca é uma doença de etiologia multifatorial. Os principais fatores de risco associados ao seu desenvolvimento são: o consumo de tabaco isoladamente ou associado ao álcool e a exposição à radiação solar ${ }^{2,3}$. Uma parcela representativa da população acometida pelo câncer de boca apresenta um perfil específico: gênero masculino, tabagismo e etilismo crônico, idade variando entre a $4^{\mathrm{a}}$ e $6^{\mathrm{a}}$ décadas de vida, baixo status socioeconômico e residência em área rural ${ }^{3,4,5}$.

Segundo o Instituto Nacional do Câncer (INCA) ${ }^{6}$, no ano de 2012, estimou-se uma incidência nacional de 9.990 casos de câncer da cavidade bucal em homens, correspondendo a um risco estimado de 10 casos a cada 100.000 habitantes. Em mulheres, se configuraram, segundo a mesma estimativa, 4.180 novos casos e um risco estimado de 4 a cada 100.000 mulheres. A estimativa para o estado do Rio Grande do Sul foi de 820 novos casos e para a capital, Porto Alegre, 110. Constata-se que, na Região Sul do Brasil, o câncer de cavidade oral é o $6^{\circ}$ tipo mais incidente em homens e o $15^{\circ} \mathrm{em}$ mulheres.

O diagnóstico e tratamento precoces do câncer são essenciais para se alcançar um prognóstico favorável, já que o atraso comumente leva à necessidade da opção por modalidades de tratamento radicais ou mesmo à impossibilidade de realização de qualquer abordagem ${ }^{7}$. A detecção do câncer e a consequente tomada de atitudes em estágios precoces acarreta diminuição na mortalidade, na morbidade e na mutilação decorrente da evolução da doença e do seu tratamento, bem como na redução de custos e aumento das taxas de sobrevivência ${ }^{8}$. Ao longo da última década, o câncer bucal tem apresentado uma taxa de sobrevida de 50\% em 5 anos.
Tal taxa aumenta para $90 \%$ nos casos em que as lesões são descobertas com tamanho menor que $2 \mathrm{~cm}$. Essa constatação torna o estágio clínico um fator preditivo de prognóstico da doença. Apesar da importância do diagnóstico precoce estar bem documentada, mais da metade dos pacientes com câncer bucal iniciam o tratamento quando as lesões já atingiram estágios avançados, situação em que a chance de sobrevida em 5 anos cai para menos de $40 \%{ }^{9}$. É presumível, portanto, que em pacientes com menor período de atraso no diagnóstico, um melhor prognóstico seja estimado ${ }^{10}$.

Os fatores que levam ao atraso no diagnóstico ainda não estão claramente definidos. Sabe-se que este pode ser gerado por questões inerentes aos profissionais ou aos pacientes. Sugere-se que a implementação de atividades de educação em saúde e a realização de exames clínicos periódicos incluindo a avaliação da mucosa bucal como procedimentos de rotina são algumas das medidas necessárias para reduzir o atraso no diagnóstico ${ }^{7}$. Com o intuito de facilitar o diagnóstico precoce, é importante que os fatores de risco sejam identificados e, quando possível, controlados?

A Atenção Primária à Saúde (APS) caracteriza-se por um conjunto de ações de saúde, no âmbito individual e coletivo, que abrangem a promoção e a proteção da saúde, a prevenção de agravos, o diagnóstico, o tratamento, a reabilitação e a manutenção da saúde. É desenvolvida por meio do exercício de práticas gerenciais e sanitárias democráticas e participativas, sob a forma de trabalho em equipe, dirigidas a populações de territórios bem delimitados, pelas quais assume a responsabilidade sanitária. Utiliza tecnologias de elevada complexidade e baixa densidade, que devem resolver os problemas de saúde de maior frequência e relevância em seu território ${ }^{11}$.

A APS é o contato preferencial dos usuários com os sistemas de saúde e orienta-se pelos princípios da universalidade, da acessibilidade e da coordenação do cuidado, do vínculo e continuidade, da integralidade, 
da responsabilização, da humanização, da equidade e da participação social. Além das características já citadas, considera o sujeito em sua singularidade, na complexidade, na integralidade e na inserção sociocultural e busca a promoção de sua saúde, a prevenção e tratamento de doenças e a redução de danos ou de sofrimentos que possam comprometer suas possibilidades de viver de modo saudável. Tem como fundamento, entre outros, efetivar a integralidade em seus vários aspectos, a saber: integração de ações programáticas e demanda espontânea; articulação das ações de promoção à saúde, prevenção de agravos, vigilância à saúde, tratamento e reabilitação, trabalho de forma interdisciplinar e em equipe e coordenação do cuidado na rede de serviços ${ }^{11}$

A coordenação do cuidado é um dos princípios da APS. Esse princípio pressupõe que o cirurgião-dentista atue nesse sistema no sentido de orientar usuários e encaminhá-los a níveis de assistência de maior complexidade, quando necessário, mantendo sua responsabilidade pelo acompanhamento do usuário e o seguimento do tratamento ${ }^{11}$. A continuidade do fluxo se dá com avaliação especializada nos Centros de Especialidades Odontológicas (CEO), serviço este enquadrado no segundo nível de atenção da rede ${ }^{12}$.

A partir destas constatações, é dado que o atraso da chegada do paciente aos setores de saúde de maior complexidade pode ser gerado por questões inerentes aos profissionais ou aos pacientes ${ }^{7}$. Para tanto, o objetivo deste trabalho é esclarecer, a partir da percepção dos profissionais, as possíveis razões presentes na APS que justifiquem o atraso da chegada do paciente aos setores de atenção em saúde de maior complexidade.

\section{Metodologia}

Trata-se de um estudo quantitativo, baseado na coleta de dados por questionário eletrônico estruturado.
Este trabalho é parte de um projeto de pesquisa que, além das etapas e resultados descritos no presente artigo, abrange também uma análise qualitativa, de mesmo objetivo principal, que apresentou como método de coleta de dados um Grupo Focal, composto por cirurgiões-dentistas atuantes em APS na cidade de Porto Alegre/RS e Região Metropolitana.

\section{Participantes}

O presente estudo teve como público alvo cirurgiõesdentistas atuantes em APS no estado do Rio Grande do Sul, participantes da estratégia TelessaúdeRS. Foram utilizados como critérios de inclusão estar vinculado formalmente ao Projeto Telessaúde e estar atuando em Estratégia de Saúde da Família (ESF).

Todos os cirurgiões-dentistas com o perfil descrito nos critérios de inclusão supracitados foram convidados a participar do estudo, logo, não houve necessidade de cálculo de amostra. O e-mail dos participantes foi obtido através do banco de dados do Projeto Telessaúde.

\section{Instrumentos}

O instrumento para coleta de dados do presente estudo foi um questionário estruturado, confeccionado pelos pesquisadores e hospedado na internet via software de questionários online SurveyMonkey ${ }^{\circledR}$.

\section{Coleta e Análise de Dados}

O presente estudo foi dividido em duas etapas. $\mathrm{Na}$ primeira etapa, foi enviada aos potenciais participantes uma correspondência eletrônica com esclarecimentos detalhados a respeito dos objetivos do projeto, bem como informações relacionadas ao preenchimento do questionário. Ao final desse e-mail, constava o link eletrônico correspondente ao questionário (http://www. 
surveymonkey.com/s/PQRJCWL), que possuía questões objetivas de escolha simples e de múltipla escolha. Esta fase do estudo foi desenvolvida nos meses de agosto e setembro de 2012.

Em etapa posterior, realizada em maio de 2013, o link eletrônico do questionário foi disponibilizado na página eletrônica de inscrição para o Curso EAD de Estomatologia para os municípios integrantes do TelessaúdeRS - $3^{\text {a }}$ edição, hospedada na homepage do TelessaúdeRS, para preenchimento pelos cirurgiões-dentistas.

Após o término do prazo de recebimento dos questionários via e-mail e do término do período de inscrições do curso citado acima, os dados, armazenados no item "Análise de Resultados" do software de questionários online SurveyMonkey ${ }^{\circledR}$, foram tabulados em uma planilha de Excel (Microsoft ${ }^{\circledR}$ ) para serem apresentados através de suas frequências absolutas.

\section{Aspectos Éticos}

O presente estudo foi submetido ao Comitê de Ética em Pesquisa do Grupo Hospitalar Conceição (CEP-GHC) e aprovado pelo parecer de número 25711, de 09 de maio de 2012.

No acesso ao link eletrônico enviado do questionário, previamente à visualização das perguntas, encontravase disponível o Termo de Consentimento Livre e Esclarecido - TCLE. Somente após visualização e resposta ao TCLE o profissional acessava o questionário. Os indivíduos tinham a liberdade de recusar-se a participar do estudo ou retirar seu consentimento em qualquer fase da pesquisa. As respostas ao questionário não apresentavam a possibilidade de identificação de autoria, logo, todos os questionários preenchidos são anônimos.

Os resultados do presente trabalho serão utilizados somente para esse estudo e ficarão armazenados durante 5 anos. Após esse período, serão totalmente excluídos. Estes cuidados minimizam os riscos decor- rentes da divulgação inadvertida das informações fornecidas pelos participantes.

Cabe ressaltar que a participação neste estudo não apresenta riscos aos participantes e possui como benefícios uma maior compreensão sobre as possíveis razões presentes em APS que possam justificar atraso no diagnóstico de pacientes com câncer bucal.

\section{Resultados}

Na primeira etapa, o convite para participar do presente estudo consistiu em um e-mail contendo uma mensagem explicativa e o link para o questionário eletrônico, que estava hospedado no software de questionários online SurveyMonkey ${ }^{\circledR}$. Esse convite foi enviado para 276 cirurgiões-dentistas atuantes em Estratégia de Saúde da Família no estado do Rio Grande do Sul, participantes do Projeto TelessaúdeRS.

O convite foi enviado inicialmente em agosto de 2012 e, após o período de 1 (um) mês, foram obtidas 17 respostas ao questionário. Devido à baixa adesão, optou-se por reenviar o convite para todos os participantes; o reenvio ocorreu em setembro de 2012. Como não era possível identificar quais os indivíduos responderam ao questionário após o primeiro envio, no corpo do segundo e-mail foi destacado que os convidados que já haviam respondido não deveriam fazê-lo novamente, para evitar a interpretação equivocada dos dados. Na segunda etapa, somaram-se às 17 participações iniciais novas 20 respostas, totalizando 37 participantes $(n=37)$ que responderam ao questionário (13,4\% da amostra inicial).

No intuito de aumentar a representatividade da amostra, durante dois decêndios do mês de maio de 2013, o link eletrônico para o questionário foi disponibilizado na página de inscrições para o Curso EAD de Estomatologia para os municípios integrantes do TelessaúdeRS - $3^{a}$ edição, hospedada na homepage do TelessaúdeRS, para 
preenchimento pelos cirurgiões-dentistas, configurando a segunda etapa de coleta de respostas.

Após o final da segunda etapa de coleta, obtevese 76 questionários iniciados. A primeira das dez perguntas do questionário consistia na apresentação do Termo de Consentimento Livre e Esclarecido. Após a descrição completa do TCLE no enunciado da questão, estavam presentes as alternativas "aceito" e "não aceito". Só seria computado o questionário que tivesse como resposta a alternativa "aceito", representando concordância com o TCLE. Verificou-se que $97,4 \%(n=74)$ selecionaram essa alternativa, logo, 2,6\% $(n=2)$ dos questionários respondidos não puderam ser computados, o que resultou em uma amostra de 74 questionários: $26,8 \%$ da amostra inicial.

A segunda pergunta apresentava o seguinte questionamento: "Na sua opinião, qual o papel do dentista da APS em relação ao câncer bucal?". "Rastreamento" foi a opção escolhida por 4,1\% (n=3), 2,7\% (n=2) marcaram "Diagnóstico", 18,9\% (n=14) marcaram "Encaminhamento", 71,6\% ( $n=53)$ marcaram "Todas as anteriores" e 2,7\% ( $n=2)$ não respondeu (Gráfico I).

\section{Na sua opinião, qual o papel do dentista da APS em relação ao câncer bucal?}

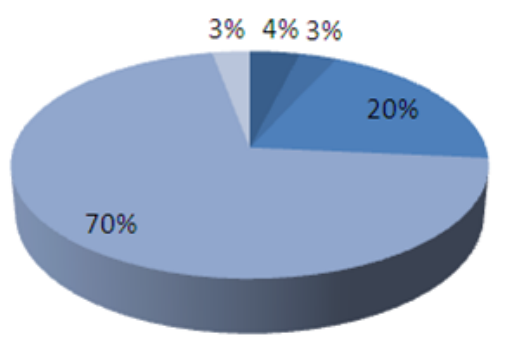

- Rastreamento a Diagnóstico Encaminhamento n Todas as anteriores não respondeu

Gráfico I: Padrão de respostas à questão de número 2

A questão seguinte permitia escolher mais de uma alternativa, o que era informado ao participante no enunciado da questão. A pergunta ("Diante de uma suspeita de câncer bucal, como você procederia?") teve o seguinte perfil de respostas: $44,6 \%(n=33)$ realizam biópsia (dentro das possibilidades estruturais e técnicas), 62,2\% ( $n=46)$ encaminham ao especialista do CEO, 40,5\% ( $n=30)$ encaminham às universidades, $4,1 \%(n=3)$ não sabem como proceder, $4,1 \%(n=3)$ encaminham o paciente ao setor de Emergência e 2,7\% ( $n=2)$ não responderam.

A pergunta de número 4 questionou: “Diante da confirmação de um caso de câncer bucal, como você procederia?". Dezenove por cento $(n=14)$ informariam ao paciente que neste momento o caso é de responsabilidade do serviço especializado e $77 \%(n=57)$ acompanhariam o paciente concomitantemente ao serviço especializado; 4,1\% (n=3) não responderam.

A questão seguinte trazia o questionamento "Como é o fluxo do paciente com suspeita de câncer de boca?". Cerca de 93\% ( $n=69)$ marcaram a alternativa "O paciente é captado em um primeiro momento no nível primário de atenção em saúde e, então, encaminhado aos demais níveis de atenção", 1,4\% $(n=1)$ marcou a alternativa “O paciente é captado diretamente no nível secundário de atenção em saúde", 1,4\% (n=1) marcou a alternativa “O paciente é captado diretamente no nível terciário de atenção em saúde" e 4,1\% (n=3) não responderam.

Na pergunta de número 6 ("Você imagina que o câncer de boca possa ser diagnosticado precocemente?"), 95,9\% $(\mathrm{n}=71)$ dos participantes marcaram a alternativa "Sim". As duas outras alternativas ("Não, o câncer só pode ser diagnosticado em estágios avançados" e "Não sei") não receberam marcações e 4,1\% (n=3) não responderam.

Quando questionados, na questão de número 7, da seguinte maneira: "Supondo que houvesse atraso no diagnóstico ou no atendimento do paciente portador desta doença no serviço secundário, quais razões você imagina que estejam envolvidas?", 5,4\% (n=4) responderam "Falha do profissional (exame clínico deficiente ou falta de familiaridade com este tipo de lesão)", 2,7\% $(n=2)$ responderam "Falha do paciente (auto-percepção deficiente)", 6,8\% $(n=5)$ responderam "Falta de informação (do paciente e do profissional)", 23\% (n=17) responderam "Falha da rede de saúde (não há serviços 
de saúde para referência)" e 58,1\% (n=43) responderam que todas as alternativas anteriores estavam corretas. Três participantes (4,1\%) não responderam (Gráfico II).

\section{Supondo que houvesse atraso no diagnóstico ou no atendimento do paciente portador desta doença no serviço secundário, quais razões você imagina que estejam envolvidas?}

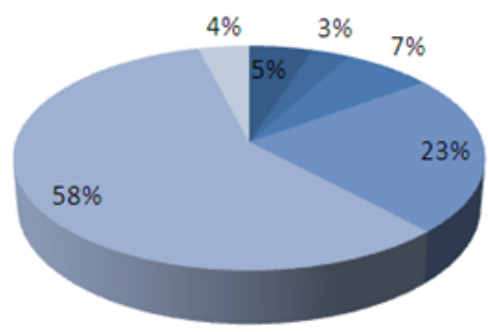

- Falha do profissional

- Falha do paciente

- Falta de informação

- Falha na rede de saúde

n Todas as anteriores

nna Na respondeu

Gráfico II: Padrão de respostas à questão de número 7.

A questão de número 8 permitia escolher mais de uma alternativa, o que era informado ao participante no enunciado. A pergunta ("Você acha que os profissionais envolvidos na equipe, além do cirurgião dentista, poderiam contribuir de alguma forma na questão do câncer bucal? Como?") teve o seguinte perfil de respostas: 14,9\% $(\mathrm{n}=11)$ de marcações na alternativa "Sim, realizando diagnóstico."; 82,4\% (n=61) de marcações na alternativa "Sim, à suspeita de lesões de boca, procurar atuar interdisciplinarmente em conjunto com o cirurgião-dentista."; 75,7\% ( $n=56)$ na alternativa "Sim, realizando educação em saúde." e nenhuma marcação (0\%) na alternativa “Não, o câncer de boca é uma temática a ser discutida apenas pelo cirurgião-dentista."; 6,8\% (n=5) não responderam.

$\mathrm{Na}$ questão de número 9, foi perguntado aos participantes se estes se consideravam aptos para realizar rastreamento e diagnóstico em câncer bucal. "Sim, conheço claramente os sinais e sintomas da doença." foi a alternativa escolhida por $10,8 \%(n=8)$ dos participantes; $71,6 \%(n=53)$ responderam "Sim, mas seriam importantes capacitações." e 12,2\% (n=9) responderam "Não, meus conhecimentos não me dão segurança para isto". Quatro participantes $(5,4 \%)$ não responderam (Gráfico III).

\section{Você se considera apto para realizar rastreamento e diagnóstico em câncer bucal?}

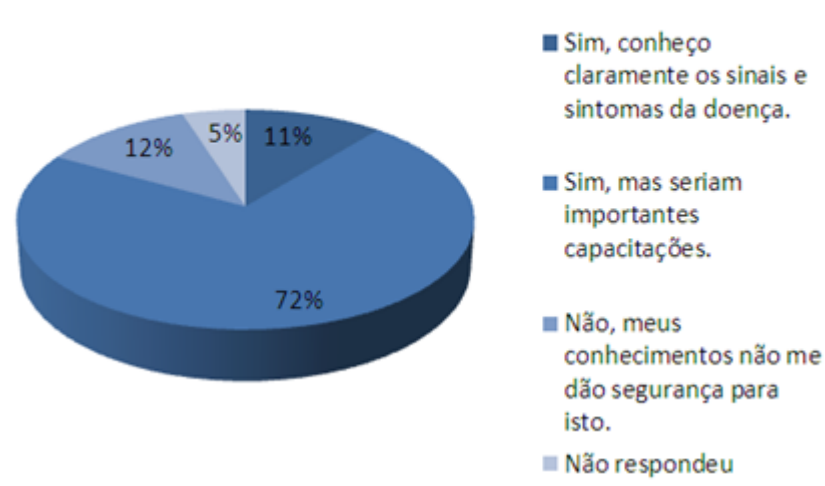

Gráfico III: Padrão de respostas à questão de número 9.

A última pergunta interrogou aos cirurgiões-dentistas se na coordenadoria regional de saúde do município em que atuam existe Centro de Especialidades Odontológicas. "Sim" foi a resposta em 33,8\% (n=25) dos casos, "Não" em $52,7 \%(n=39)$ do casos, $8,1 \%(n=6)$ responderam que não sabem e 5,4\% (n=4) não responderam (Gráfico IV).

\section{$\mathrm{Na}$ coordenadoria regional de saúde do seu município, existe Centro de Especialidades Odontológicas?}

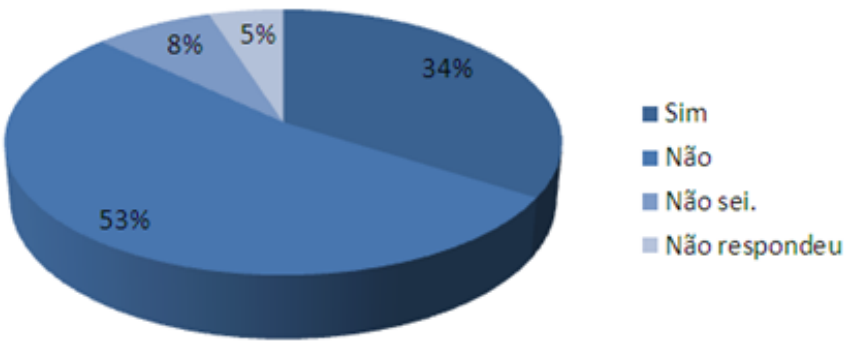

Gráfico IV: Padrão de respostas à questão de número 10.

\section{Discussão}

Primeiramente, é válido ressaltar que a taxa de respostas ao questionário, $26,8 \%$ da amostra inicial, permaneceu aquém da média encontrada na literatura. Em uma busca de artigos que avaliaram a percepção dos dentistas a respeito do câncer de boca por meio de questionário via correspondência, realizada na base de dados PUBMED, foi en- 
contrada uma taxa de retorno dos questionários na faixa de $14 \%$ a $55,2 \%$, sendo a média $37,9 \% 13,14,15,16$. Sugere-se que, apesar de o método de envio eletrônico ser mais ágil e prático do que a correspondência convencional, tanto para os participantes como para os pesquisadores, pode gerar desconfiança e falta de credibilidade, relacionadas à sua impessoalidade.

Segundo as Diretrizes da Política Nacional de Saúde Bucal $^{17}$, à APS compete assumir a responsabilidade pela detecção das necessidades, providenciar os encaminhamentos requeridos em cada caso e monitorar a evolução da reabilitação, bem como acompanhar e manter a reabilitação no período pós-tratamento. Tratando-se especificamente de câncer bucal, é preconizado que se ofereça oportunidades de identificação de lesões bucais (busca ativa), através de visitas domiciliares ou em momentos de campanhas específicas, consolidando a lógica do rastreamento. Além disso, o diagnóstico precoce de lesões de mucosa e do câncer de boca deve ser uma ação desenvolvida sistematicamente pelas equipes de saúde bucal na atenção básica12. O perfil de respostas ao questionamento sobre as atribuições do dentista de APS em relação ao câncer bucal indica que o profissional alvo dessa pesquisa compreende seu papel no cuidado relacionado a essa patologia, com $71,6 \%$ das respostas na alternativa que melhor o define. Essa constatação ainda é ratificada por $93 \%$ de respostas na alternativa mais adequada à pergunta relacionada ao fluxo que o paciente deve percorrer no sistema, a qual indica compreensão sobre o papel da APS no fluxo do paciente com câncer de boca na Rede de Atenção à Saúde (RAS).

A questão relacionada ao destino do encaminhamento revela que apenas $62,2 \%$, diante da suspeita de um diagnóstico de câncer bucal, encaminham o paciente a um CEO, sendo este o fluxo considerado ideal, segundo o Caderno de Atenção Básica n $17^{12}$. Esse perfil de respostas pode estar traduzindo duas hipóteses: a primeira, a falta de uma retaguarda adequada no que concerne à atenção secundária, ou seja, uma rede deficiente e carente de Centros de Especialidades Odontológicas; a segunda, falta de conhecimentos sobre o fluxo ideal, não sabendo o dentista para quem encaminhar. A segunda hipótese, no entanto, é desencorajada pela baixa porcentagem de respostas da alternativa "não sabe como proceder" (4,1\%). Além disso, fortalecem a primeira hipótese as respostas encontradas nas questões sobre os possíveis motivos de atraso no diagnóstico e sobre a existência de CEO na Coordenadoria Regional de Saúde na qual o profissional atua.

Na pergunta sobre os possíveis motivos de atraso no diagnóstico, a resposta mais selecionada foi "todas as anteriores" (falha do profissional - exame clínico deficiente ou falta de familiaridade com esse tipo de lesão; falha do paciente - auto-percepção deficiente; falta de informação - do paciente e do profissional e falha da rede de saúde - não há serviços de saúde para referência) como possíveis razões que justificariam uma situação de atraso no diagnóstico. A segunda resposta mais selecionada foi "falha da rede de saúde (não há serviços de saúde para referência)", sendo considerada esta, de acordo com a somatória das respostas, uma importante situação identificada pelos participantes. Já na pergunta sobre a existência de Centro de Especialidades Odontológicas na Coordenadoria Regional de Saúde na qual o profissional atua, 52,7\% dos dentistas indicaram que não há CEO de referência para sua Unidade de Saúde, evidenciando a deficiência da rede.

Em consonância com a percepção apontada pelo estudo, estão os atuais indicadores de saúde bucal relacionados à atenção especializada no estado do Rio Grande do Sul. Contrastando com a realidade encontrada no estado de Minas Gerais, que possui aproximadamente 1 CEO para cada 240 mil habitantes, no estado do Paraná (1 CEO para cada 208 mil habitantes) e em Santa Catarina (1 CEO para cada 159 mil habitantes), o 
Rio Grande do Sul conta com 1 Centro de Especialidade Odontológicas para cada 550 mil habitantes, totalizando apenas 20 CEO em todo o estado ${ }^{18}$.

Definido por Starfield ${ }^{19}$ como um dos princípios ordenadores da APS, a coordenação do cuidado, a qual tem sua conceituação relacionada à capacidade do serviço em garantir a continuidade da atenção, o seguimento do usuário no sistema ou a garantia da referência a outros níveis de atenção quando necessário ${ }^{31}$ mantendo a corresponsabilização sobre o caso, parece estar legitimada no processo de trabalho dos participantes do presente estudo. Com $77 \%$ de respostas na alternativa "acompanha o paciente concomitantemente ao serviço especializado", após serem questionados sobre qual a conduta diante da confirmação de um caso de câncer bucal, os dentistas participantes mostraram familiarização com a lógica de trabalho que valoriza o vínculo e a responsabilização com a população adscrita, o que garante a continuidade das ações de saúde e a longitudinalidade do cuidado ${ }^{12}$.

Em relação ao conhecimento dos participantes sobre aspectos inerentes ao diagnóstico clínico da patologia, o perfil de respostas da questão relacionada à fase de diagnóstico demonstra que o conceito base de diagnóstico precoce está perpetuado na amostra, entretanto, as perguntas sobre os possíveis motivos de atraso no diagnóstico e sobre autopercepção de aptidão para diagnóstico e rastreamento indicam alguma carência de embasamento para um manejo seguro do câncer bucal.

As repostas à pergunta sobre os possíveis motivos de atraso no diagnóstico sugerem uma realidade de informação insuficiente, expressa pelos 58,1\% de escolha na alternativa que elege a falha do profissional (exame clínico deficiente ou falta de familiaridade com este tipo de lesão) como uma das razões de um possível atraso no diagnóstico de câncer bucal. Já as respostas para a pergunta que aborda autopercepção de aptidão para diagnóstico, apontam para uma sensação de insegurança nos participantes: ao serem questionados sobre estarem preparados para realizar rastreamento e diagnóstico em câncer bucal, 71,6\% escolheram a alternativa "Sim, mas seriam importantes capacitações" e $12,2 \%$ a alternativa "Não, meus conhecimentos não me dão segurança para isto". Esse achado está em sincronia com Falcão et al. ${ }^{20}$, o qual verificou que $69,5 \%$ dos 240 dentistas participantes consideravam baixo seu nível de confiança para realizar procedimentos de diagnóstico de câncer bucal.

Realizar o cuidado em equipe e atuar de maneira interdisciplinar são fundamentos básicos no processo de trabalho em APS, reforçados na lógica da Estratégia de Saúde da Família ${ }^{11}$. Essa estratégia, que objetiva primordialmente a integralidade do cuidado, parece estar bem estabelecida na concepção dos participantes e foi traduzida de maneira evidente no padrão de respostas na questão relacionada ao papel da equipe na abordagem do câncer bucal.

É válido ressaltar que, para considerar tradução real do atual cenário estudado, a amostra deveria ser mais numerosa, sendo sua representatividade um viés desta pesquisa.

\section{Conclusões}

O presente estudo sugere como possíveis razões que justifiquem o atraso da chegada do paciente com câncer bucal aos setores de maior complexidade de atenção em saúde, sob a óptica dos cirurgiões-dentistas atuantes em APS no estado do Rio Grande do Sul, a deficiência da rede de atenção, com notável carência de serviços especializados em odontologia (CEO) e a necessidade de mais capacitações relacionadas à patologia em foco. É válido ressaltar que o estudo não teve como objetivo investigar as razões do atraso inerentes ao paciente e sim relacionadas às etapas de responsabilidade do profissional/serviço. 
Os resultados apontam para uma compreensão elevada dos participantes acerca dos princípios e lógica de funcionamento da Atenção Primária à Saúde, das suas atribuições em APS relacionadas ao câncer bucal, do papel da APS na Rede de Atenção à Saúde e dos fluxos da RAS.

Estudos que apresentam como meio de coleta questionários eletrônicos devem contar com estratégias que estimulem maior adesão dos potenciais participantes.

\section{Referências}

1. Neville NW, Day TA. Oral cancer and precancerous lesions. CA Cancer J Clin. 2002;52:195-215.

2. Napier SS, Speight PM. Natural history of potentially malignant oral lesions and conditions: an overview of the literature. J Oral Pathol Med. 2008;37:1-10.

3. Scully C, Bagan JV. Oral squamous cell carcinoma: overview of current understanding of aetiopathogenesis and clinical implications. Oral Diseases. 2009;15:388-99.

4. Attar E, Subhojit D, Hablas A, Seifeldin I, Ramadn M, rozek L, et al. Head and neck cancer in a developing country: A population-based perspective across 8 years. Oral Oncology 2010;46:591-96.

5. Zini A, Czerninski R, Sgan-cohen HD. Oral cancer over four decades: epidemiology, trends, histology, and survival by anatomical sites. J Oral Pathol Med. 2010;39:299-305.

6. Instituto Nacional do Câncer. Incidência de Câncer no Brasil em 2012. Disponível em: < http://www.inca.gov.br/estimativa/2012/>. Acesso: 10/08/2013

7. Onizawa K, Nishihara K, Yamagata K, Yusa H, Yanagawa T, Yoshida H. Factors associated with diagnostic delay of oral squamous cell carcinoma. Oral Oncol. 2005;39(8):781-9.
8. Scott SE, Grunfeld EA, Mcgurk M. Patient's delay in oral cancer: a systematic review. Community Dent Oral Epidemiol. 2006;34(5):337-43.

9. Scott SE, Grunfeld EA, Mcgurk M. The idiosyncratic relationship betweendiagnostic delay and stage of oral squamous cell carcinoma. Oral Oncol. 2005;41(4):396-403.

10. Gomez I, Seoane J, Varela-Centelles P, Diz P, Takkouche B. Is diagnostic delay related to advanced-stage oral cancer? A meta-analysis. Eur J Oral Sci. 2009;117:541-46.

11. Ministério da Saúde (Brasil). Portaria $n^{\circ} 2488$ de 21 de outubro de 2011. Disponível em: <http://www.saude.mt.gov.br/upload/legislacao/2488-[5046-041111-SES-MT].pdf>. Acesso: 5/10/2012

12. Ministério da Saúde (Brasil). Caderno de Atenção Básica n 17. 2008. Disponível em: <http://bvsms.saude.gov.br/bvs/publicacoes/saude_bucal.pdf >. Acesso: $24 / 10 / 2012$

13. Alonge OK, Narendran S. Opinions about oral cancer prevention and early detection among dentists practicing along the Texas-Mexico border. Oral Diseases. 2003;9:41-5.

14. Clovis JB, Horowitz AM, Poel DH. Oral and pharyngeal cancer: knowledge and opinions of dentists in British Columbia and Nova Scotia. J Can Dent Assoc. 2002;68(7):415-20.

15. Hertrampf K, Wenz HJ, Koller M, Wiltfang J. Comparing dentists' and the public's awareness about oral cancer in a community-based study in Northern Germany. Journal of Carnio-Maxillo-Facial Surgey. 2010;1-5.

16. Jornet PL, Alonso FC, Miñano FM. Knowledge and attitudes about oral câncer among dentists in Spain. Journal Evaluation in Clinical Practice. 2010;16:129-33.

17. Ministério da Saúde (Brasil). Diretrizes da Política Nacional de Saúde Bucal. Brasília: 2004. Disponível em: <http://conselho.saude.gov.br/web_comissoes/cisb/doc/ politica_nacional.pdf $>$. Acesso: 1/10/2012

18. Ministério da Saúde (Brasil). Sala de Apoio à Gestão Estratégica do Ministério da Saúde. Disponível em: <http://189.28.128.178/sage/>. Acesso: 1/10/2012

19. Starfield, B. Atenção Primária Equilíbrio entre Necessidades de Saúde, Serviços e Tecnologia. Unesco Brasil: 2002.

20. Falcão MML, Alves TDB, Freitas VS, Coelho TCB. Conhecimento dos cirurgiõesdentistas em relação ao câncer bucal. RGO, Porto Alegre. 2010;58(1):27-33. 